\title{
Fritz Lang in Hollywood
}

Das mythische Eigene und die Fremdheit des Mythos

Fritz Lang à Hollywood: familiarité et étrangeté du mythe

Fritz Lang in Hollywood: Familiarity and Strangeness of Myth

\section{Stephanie Wodianka}

\section{(2) OpenEdition}

\section{Journals}

Édition électronique

URL : http://journals.openedition.org/ceg/4793

DOI : $10.4000 /$ ceg.4793

ISSN : 2605-8359

\section{Éditeur}

Presses Universitaires de Provence

\section{Édition imprimée}

Date de publication : 2 mai 2019

Pagination : 189-200

ISBN : 979-10-320-0214-8

ISSN : 0751-4239

\section{Référence électronique}

Stephanie Wodianka, „Fritz Lang in Hollywood“, Cahiers d'Études Germaniques [Online], 76 | 2019, Online erschienen am: 02 November 2020, abgerufen am 25 Januar 2021. URL: http://

journals.openedition.org/ceg/4793 ; DOI: https://doi.org/10.4000/ceg.4793 


\section{Fritz Lang in Hollywood \\ Das mythische Eigene und die Fremdheit des Mythos}

Stephanie WODIANKA

Universität Rostock

\section{Die Nibelungen: befremdlich-unvertraute Inszenierung des Eigenen als Exotisch-Anderes}

Fragt man nach dem Verhältnis Fritz Langs zum Mythos, so mag zunächst sein Filmschaffen vor seiner Emigration im Jahr 1939 in den Sinn kommen. Fritz Lang, 1890 in Wien geboren und 1976 in Kalifornien gestorben, schuf 1924 mit Die Nibelungen $^{1}$ einen zweiteiligen Stummfilm, der den modernen Mythos Mittelalter erzählt. Der Film wurde mit Spannung vom Publikum erwartet, die Veröffentlichung des von Fritz Langs Ehefrau Thea von Harbou verfassten Nibelungenbuchs 1923 sowie Fritz Langs Pressebeiträge steigerten die mediale und öffentliche Erwartungshaltung auf das Werk, das den Mythos Mittelalter im zum Mythos verklärten Medium Film auf die Leinwände bringen sollte: „Er [der Film] hat uns das Reich des Zauberhaften erschlossen“, so schreibt Lang kurz vor der Premiere am 1. Januar 1924.

\footnotetext{
Es liegt über ihm etwas vom Mystischen der Schöpfung. Ein Wille sagt: Es werde! Und siehe da, es wird! Es wird durch das modernste aller Mittel: vollendete Technik. Es gibt für den Film weder Zeit- noch Raumbegriffe. Es ist durch ihn möglich, da zu gleicher Zeit, in allen fünf Weltteilen das gleiche Bild von Millionen verschiedener Menschen lebendig wird. ${ }^{2}$
}

In Die Nibelungen sollten Mittelalter und Moderne zum mythischen Amalgam verschmelzen und zugleich den Mythos Kino mitbegründen.

Inwiefern Fritz Langs Nibelungen auch unter dem Zeichen völkischer und deutschtümelnder Selbstbegründung stehen, ist zumindest in der neueren Forschung umstritten. Einerseits zeigen einige Äußerungen des Filmregisseurs, dass er das mittelhochdeutsche Nibelungenlied als deutsche Gründungserzählung interpretierte. Der doppeldeutige Untertitel „Dem Deutschen Volke zu eigen“ erklärt entweder die Nibelungen zum Eigentum des deutschen Volkes, oder aber er lässt sich deuten als Widmung des Filmes, der dem deutschen Volke zu Eigen gemacht werden

1. Die Nibelungen. Regie: Fritz Lang. Buch: Fritz Lang, Thea von Harbou. Deutschland 1924.

2. Fritz Lang, „Stilwille im Film“, Jugend 3, 1924. Zit. nach Fred Gehler, Ullrich Kasten (Hrsg.), Fritz Lang. Die Stimme von Metropolis, Berlin, Henschel, 1990, S. 162. 
soll. Er legt auch das Selbstverständnis des Films als Medium nahe, selbst Teil des identitätsbegründenden kulturellen Gedächtnisses der Deutschen zu sein, die Lang zu neuer nationaler Stärke führen wollte: „Nach der Niederlage des Ersten Weltkrieges wollte ich den Deutschen dadurch, dass ich ihre berühmte Sage verfilme, wieder ein gewisses Nationalbewusstsein zurückgeben“, so Fritz Lang noch in einem Interview 1971. ${ }^{3}$ Und seine Frau und Drehbuchautorin Thea von Harbou belegt diese Vermutung, wenn sie im Premierenheft schreibt: „Diesem deutschen Volke soll der Nibelungen-Film zum Sänger, zum erzählenden Dichter seiner selbst werden." Der Film wird zum neuen Minnesang, der vom Volk und zum Volk dichtet. Das Kinoerlebnis wird zum Gedächtnisritual, das deutsche Gründungswerte in scheinbarer Evidenz erfahrbar macht.

Andererseits zeigt eine tiefergehende Analyse des Nibelungenfilms, dass Fritz Lang das Mittelalter nicht ungebrochen als das deutsche Eigene inszenierte. Denn das Mittelalter Langs ist vor allem eins: befremdlich, unvertraut. Dem Rezipienten wird es äußerst schwergemacht, sich mit diesem Mittelalter zu identifizieren. Statt sein Publikum in der filmischen Illusion zu wiegen, im Mittelalter zu Hause zu sein, nutzt Lang die ihm zur Verfügung stehenden filmischen Möglichkeiten, das Mittelalter als eigene Fremde vorzuführen. Langs filmische Nibelungenerzählung ist in diesem Freudschen Sinne ,unheimlich $^{4}$ und zeigt die Konfrontation mit dem unvertraut gewordenen Eigenen. Die Nibelungen werden, wie Silke Hoklas in ihrer einschlägigen Studie gezeigt hat $^{5}$, zum Ort des Kulturkontakts, und das nicht nur, weil sie für den internationalen Kinomarkt bestimmt sind und nur bei Zuspruch durch ein internationales Publikum die immensen Herstellungskosten von über 8 Millionen Reichsmark gedeckt werden konnten - ein eindimensional nationalistischer Film wäre kaum $\mathrm{zu}$ vermarkten gewesen. Fritz Langs Nibelungen sind auch deshalb eine Kulturkontaktzone von Mittelalter und Moderne, weil sie die Alterität des Mittelalters imaginativ vergegenwärtigen durch das vertraute Andere. ${ }^{6}$ Fritz Lang hat seine Inszenierungen des Mittelalters durch den Rückgriff auf Archive des exotisch und ethnisch Anderen gestaltet. Prägnantestes Beispiel dafür ist sicherlich die Inszenierung König Etzels im zweiten Teil der Nibelungen, bei der Lang auf eine Melange von Elementen verschiedenster außereuropäischer (asiatischer bis

3. „Es soll, ruhig schauend, sich beschenken lassen, empfangend erleben und damit neu gewinnen, was ihm, dem Volke als Ganzes, nur noch blasse Erinnerung ist: das Hohelied von bedingungsloser Treue.“ (Thea von Harbou, Programmheft anlässlich der Premiere). Das Paradox, dass dieses Selbstbewusstsein ausgerechnet durch einen Untergangsmythos begründet werden sollte, lässt sich dadurch auflösen, dass dieser zum Appell wird, genau jenem drohenden Untergang entgegenzuwirken. Die Dolchstoßlegende konnte nach dem Ersten Weltkrieg als mythische Renarration des Nibelungenliedes über Treue und Verrat gelten, ihr identifikatorisches Potential gewinnt sie durch den Aufruf zur Verhinderung zukünftigen kulturellen Untergangs im Gedächtnis dieser Erfahrung.

4. Sigmund Freud, „Das Unheimliche“, in ders., Anna Freud (Hrsg.), Gesammelte Werke. Chronologisch geordnet. Bd. 12, Frankfurt a. M., Fischer Taschenbuch-Verlag, 1999 [1919], S. 227-278.

5. Silke Hoklas, Eigene Fremde. Konstruktionen des Mittelalters in den Stummfilmen von Fritz Lang, Dissertation Universität Rostock, 2015, S. 352-590.

6. Zur Konstruktion von Alterität durch Differenzierung und Distanzierung s. Andrea Polaschegg, Der andere Orientalismus. Regeln deutsch-morgenländischer Imagination im 19. Jahrhundert, Berlin/ New York, de Gruyter, 2005, S. 41- 49. 
indianischer) Kulturen setzt, um innerhalb des mittelalterlichen Settings eine Hierarchie des Fremden zu erzeugen. ${ }^{7}$ Er übernimmt dabei Darstellungskonventionen des europäischen Kolonial- und Orientalismusdiskurses, die beim Publikum Wiedererkennungseffekte auslösen. ${ }^{8}$ Das internationale, ,moderne“ Publikum des monumentalen Nibelungen-Films sah sich somit vereint in interkultureller Differenz zum Mittelalter. Das national-völkische, plurimediale framing der Premiere in Deutschland, das durch Interviews und schriftliche Beiträge Langs und seiner Frau Thea von Harbou markiert wurde, machte den Film aber zugleich rezipierbar als mythische Gründungserzählung. ${ }^{9}$

\section{Filmschaffen in Exil: Erwartungshaltungen und Selbstanspruch}

Fritz Lang entschloss sich 1933 spontan zur Emigration ${ }^{10}$ - er selbst stellte später seinen Entschluss in unmittelbaren Zusammenhang mit dem Angebot Goebbels, ihn zum Leiter des Deutschen Films zu machen. ${ }^{11}$ Er war angeblich ohne Geld in den Nachtzug nach Paris gestiegen, und schon 1934 realisierte er mit Erich Pommer in Frankreich den Tonfilm Liliom und wanderte noch im selben Jahr in die USA aus. Zwei Jahre später entstand sein erstes Werk im amerikanischen Exil: Der Tonfilm Fury aus dem Jahr 1936, der im Zentrum dieses Beitrages stehen soll. Langs Exilwerk steht unter neuen Vorzeichen: Die Erfahrungen im nationalsozialistischen Deutschland, aber auch die veränderten Produktionsbedingungen und gesellschaftspolitischen Kontexte in Hollywood haben zu einer ästhetischen und vordergründig auch thematischen Änderung seines Filmschaffens geführt. Fritz Lang war zwar in den USA bereits bekannt (insbesondere durch Die Nibelungen), dennoch musste er sich - als Exilant noch mehr als alle anderen - den Erwartungen der Hollywood-

7. S. dazu ausführlich Hoklas, Eigene Fremde, S. 533-538. Zur Frage der ,jüdischen“ Gestaltung der Figuren s. die differenzierte Sicht bei David J. Levin, Richard Wagner, Fritz Lang, and the Nibelungen. The Dramaturgy of Disavowal, Princeton University Press, 1999, S. 8-12.

8. „Langes Mittelalter ist durch die verschiedenen Mittelalterbilder, die es in sich vereint, zutiefst hybrid. Auf der Produktionsebene ist das ein interessanter Versuch, die Pluralität der breiten öffentlichen wie akademischen Nibelungen- und Mittelalterrezeption einzufangen, auf der Rezeptionsseite jedoch lässt sich vermehrt beobachten, dass nicht diese Pluralität wahrgenommen wird, sondern stattdessen lediglich für ein umso breiteres Publikum jeweils etwas Identifikatorisches dabei ist. Das hybride Mittelalterbild der Nibelungen bietet dadurch für jeden etwas, doch zugleich für niemanden eine durchgängige Interpretation." (Hoklas, Eigene Fremde, S. 596)

9. „Er setzt einerseits auf einzelne Motive, Traditionen und Tableaus, die im kulturellen Gedächtnis fest verankert sind, und andererseits auf eine ausgeprägte Exotisierung des Mittelalters, die sich als Rückübertragung der gängigen Historisierung des Exotischen auf die eigene Vergangenheit beschreiben lässt [...].“Ibid., S. 682.

10. Zur Schilderung seines Entschlusses, ins Exil zu gehen, s. Norbert Grob, Fritz Lang. ,Ich bin ein Augenmensch: Die Biographie. Berlin, Propyläen, 2014, S. 186-189.

11. Nach dem Publikumserfolg mit Die Nibelungen gelang Fritz Lang 1927 ein weiterer erfolgreicher Stummfilm mit dem Science-Fiction-Klassiker Metropolis, bevor er mit $M$ seinen Durchbruch auf dem Feld des Tonfilms feierte und dessen ästhetische Potentiale nutzte - unvergessen ist uns die gepfiffene Melodie, an der der blinde Luftballonverkäufer den Kindermörder identifiziert. 
Produktion im Sinne der Maxime „more oft he same“ fügen. ${ }^{12}$ Seine kritische Haltung zur Standardisierung des Hollywood-Erfolgsfilms hatte er mehrfach schon vor seiner Emigration zum Ausdruck gebracht. ${ }^{13}$ Er sieht die Rolle des Kinos nicht nur darin, Erwartungshaltungen des Publikums zu befriedigen, sondern auch darin, prägend und wirksam zu werden im Sinne einer kulturellen Orientierung, die nicht nur affirmativ sein darf:

Unser Publikum bevorzugt eine solche affirmative Story gegenüber der Tragödie [...]. Aber ich bin sicher, dass es etwas darüber Hinausgehendes gibt, das den wirklichen kulturellen Bedürfnissen gerecht wird, die hinter den Publikumsbedürfnissen stecken. Und es ist die unvergessliche Funktion der Künstler, eine Richtschnur zu sein auf den Pfaden kultureller Entwicklung. ${ }^{14}$

Der Film Fury, ${ }^{15}$ auf den ich nun die Aufmerksamkeit lenken möchte, steht im Zeichen dieses Selbstanspruches. Lang nutzte seine Gestaltungsmöglichkeiten als Regisseur, um seiner ersten amerikanischen Produktion einen eigenen Stempel aufzudrücken, zugleich Impulse seines amerikanischen Exils aufzunehmen und anzuschließen an seinen auch in den USA bekannten Erfolg von Die Nibelungen. Dieser Anschluss verläuft über thematische und filmästhetische Brücken, aber wie hier zu zeigen ist auch ganz wesentlich über eine Mythopoiesis, die Langs Handschrift trägt.

12. „Dieses ,Erfolgsrezept ${ }^{\star}$ der idealistischen, affirmativen Unterhaltungsware wurde in der Produktionsfirma Metro-Goldwyn Mayer stets beherzigt. [...] Leichte Unterhaltungsfilme und opulent ausgestattete Musicals ließen die Gewinne an den Kinokassen steigen. Dieser wirtschaftliche Erfolg war jedoch mit einer starken Hierarchisierung des Produktionsprozesses ,erkauft ‘ worden. In keinem anderen Hollywood-Studio übte die Führungsspitze - repräsentiert durch die Produzenten - größeren Einfluss auf das künstlerische Personal aus.“ (Larissa Schütze, Fritz Lang im Exil. Filmkunst im Schatten der Politik, München, Meidenbauer, 2006, S. 48) Zu den Bedingungen und Einflüssen des Filmschaffens in Hollywood s. auch Cornelius Schnauber, Fritz Lang in Hollywood, Wien, Europaverlag, 1986.

13. „Erfolgsrezept dieses Standardfilms: Schönes Mädel - braver Bursche - Schurke (meist mit mexikanischem Einschlag, immer mit kleinem Schnurrbart!) - Gefahr - allerhöchste Gefahr Todesnot - Bumm! Krach! - Die Rettung! - Die Guten belohnt, die Bösen gestraft - Halleluja!“ (Fritz Lang, „Was ich in Amerika sah“, Film-Kurier, Nr. 294, 13. Dezember 1924. Zit. nach Gehler, Ullich, Stimme von Metropolis, S. 213.)

14. „Unser Publikum bevorzugt eine solche affirmative Story gegenüber der Tragödie, und ich denke, dass das Publikum damit Recht hat. Aber ich bin sicher, dass es etwas darüber Hinausgehendes gibt, das den wirklichen kulturellen Bedürfnissen gerecht wird, die hinter den Publikumsbedürfnissen stecken. Und es ist die unvergessliche Funktion der Künstler, eine Richtschnur zu sein auf den Pfaden kultureller Entwicklung.“ [Übersetzung S.W.] - Engl. Original: „Our audience prefers such a [affirmative] story to tragedy, and I think the audiance is right in its preference. But I am sure there is something further which will answer the real cultural needs which lie behind the audience's demands. And it is the immemorial function of artists to lead the way along the paths of cultural development." (Thomas Strack, „Fritz Lang und das Exil. Rekonstruktion einer Erfahrung mit dem amerikanischen Film“, in Willi Jasper, Joachim H. Knoll (Hrsg.), Preußens Himmel breitet seine Sterne... Festschrift zum 60. Geburtstag von Julius H. Schoeps, Hildesheim, Olms, 2002, S. 410.)

15. Fury. Regie: Fritz Lang. Buch: Fritz Lang, Bartlett Cormack (nach einer Erzählung von Norman Krasna). USA 1936. 


\section{The Fury: Roosevelts regular guy und die Zerbrechlichkeit demokratischer Ordnung}

Die Film-Idee zu Fury, die Fritz Lang auf der Basis eines zehnseitigen Entwurfs präsentierte, geht auf ein wahres Ereignis des Jahres 1933 in San José zurück ${ }^{16}$, das die Aufmerksamkeit von Presse und Öffentlichkeit auf sich gezogen hatte: Eine aufgebrachte Menge hatte das Gefängnis gestürmt, zwei des Kidnapping verdächtigte Männer entführt und durch Hängen gelyncht. Die US-amerikanischen Drehbuchautoren Norman Krasna und Joseph L. Mankiewicz boten Fritz Lang an, aus ihrem zehnseitigen Treatment mit dem Titel Mob rule einen Film zu machen. Die Zusammenarbeit erwies sich als konfliktuös, aber im Ergebnis produktiv. Fritz Lang sah von Beginn an die Tiefe des Sujets, bei dem es darauf ankam, nicht der Simplizität der Hollywood-Industrie zu erliegen. ${ }^{17}$

Fury hat mehr zu bieten als ein Plädoyer gegen Lynch-Justiz - denn der Film, so ist zu zeigen, geht darüber hinaus und leistet eine Mythenkritik des zeitgenössischen Amerika. Nicht, um diese Mythen zu Ende zu bringen, sondern um ihre Fragilität aufzuzeigen und auf diese Weise für ihre notwendige Stabilisierung zu plädieren. Im Fokus steht ein ganzes Mythen-Netzwerk, das sich um die mythische Figur des Durchschnittsamerikaners „John Doe“ in den 1930er Jahren entspinnt: Es sind die normvermittelnden Erzählungen von American Dream, Gerechtigkeit, Verfassung, Roosevelts New Deal, Civilization, Freiheit, Fortschritt, die im Film ikonisch komprimiert und zugleich exemplarisch narrativ entfaltet werden. Fritz Lang wagt sich mit Fury an die Grundfeste amerikanischen Selbstverständnisses.

Dabei tut er viel dafür, dass wir in seinem Protagonisten Joe den amerikanischen regular guy John Doe wiedererkennen. Dieser steht mit seiner Verlobten Katherine vor einem Schaufenster, das eine Brautausstattung und ein eheliches Schlafzimmer dekoriert hat: Die symbolische Verwirklichung bürgerlich-anständiger Lebensführung, die zugleich Erfüllung und Domestikation ${ }^{18}$ von Bedürfnissen ist, und zu der Katherine - wie der Zuschauer durch den Dialog der beiden erfährt - Joe bereits ihr Jawort gegeben hat. Der Film baut die beiden Charaktere als Personifikationen des American Dream auf. Joe will versuchen, durch redliche Arbeit gutes Geld zu verdienen und zu sparen, um die geplante gemeinsame Wohnung zu finanzieren. Katherine gibt ihren Job vor Ort auf, um im weit entfernten Gladesbury eine besser bezahlte Arbeit anzunehmen. Das Paar nimmt die weite räumliche Trennung auf sich und stellt aktuelle Bedürfnisse hinten an, um den gemeinsamen,

16. Vgl. Schütze, Fritz Lang im Exil, S. 61f.

17. „Wenn man einen Film über Lynchmord drehen will, dann nimmt man am besten eine weiße Frau, die von einem Schwarzen vergewaltigt wird und zeigt anhand dieser Ausgangssituation, dass Lynchen trotzdem Unrecht ist. » (Zit. nach Grob, Fritz Lang, S. 219f.) Gunning sieht den Film als Teil einer Sozialtrilogie, zusammen mit You only live Once (1937) und You an Me (1938) - s. Tom Gunning, The Films of Fritz Lang. Allegories of Vision and Modernity, London, British Film Institute, 2006, S. 203-282.

18. Bereits in dieser Eingangsszene bietet der Film Bezüge zu Freuds Theorien des individuellen Triebverzichts und des Verdrängens. Diese werde im späteren Verlauf des Films auf kollektiver Ebene durch das Motiv des entfremdeten Eigenen (s.u.) aufgegriffen. 
durch aufrichtige Liebe wie materielle Prosperität konturierten Lebenstraum zu verwirklichen. Ästhetische Effekte des Tonfilms werden zudem genutzt, um den einfahrenden Zug Katherines - Symbol technischen Fortschritts und moderner Mobilität - eindrucksvoll in unmittelbarer Nähe des Filmpublikums vorbeirauschen zu lassen: Das Paar geht mit der Zeit und sieht - getragen vom Fortschrittsoptimismus des New Deal Roosevelts - einer erfüllten Zukunft entgegen.

Als Kontrastfiguren dienen nach Zugabfahrt die Brüder Joes. Sie lehnen sein anständiges Leben $\mathrm{ab}$, trinken, zocken und schlagen sich durch zwielichtige halbkriminelle Geschäfte und parasitäre Mitbewohner Joes durch. Das Streitgespräch zwischen Joe und Bruder Charly führt vor, wofür Joe einsteht: Als leistungsbereiter Durchschnittsamerikaner gehören für ihn Anstand und Wohlstand zusammen. Für Joe ist das neue Heldentum ein Heldentum der Masse, das jedem möglich ist, der sich den neuen Werten von Leistung, Anstand und Optimismus verschreibt. Gerade hier tritt Langs Referenz auf den zeitgenössischen New Deal der 30er Jahre unter Roosevelt zutage, dessen Wirtschaftsprogramm auf dieser Idee beruhte. Belohnt wird Joe wenige Wochen später durch den Erfolg einer Optionsaktie, die ihm - gemeinsam mit seinen nun bekehrten Brüdern - das Betreiben einer eigenen Tankstelle erlaubt, die für den wirtschaftlichen wie technischen Fortschritt gleichermaßen steht. Zum Unternehmer promoviert, steht ihm der Weg zur Hochzeit endlich offen, und er macht sich auf den Weg zur sehnsüchtig wartenden Katherine.

Der Filmbeginn baut somit ostentativ in Joe die mythische Figur John Doe auf, deren Lebensgeschichte als Renarration der modernen Mythen Fortschritt und American Dream erscheint. Durch diese multimythische Fundierung erreicht Fritz Lang die Fallhöhe seines Protagonisten, die den später an ihm vollzogenen Lynchmord kathartisch wirksam macht. Kein schwarzer Schuldiger, sondern ein weißer Unschuldiger wird Opfer der kollektiven Lynchtat: Auf dem Weg zu Katherine - kurz vor der Erfüllung seiner Träume - wird Joe kontrolliert und aufgrund einer unglücklichen Verkettung falscher Indizien bezichtigt, der gerade gesuchte Kidnapper zu sein. An seinem Fall wird das Kollabieren eigentlich geltender, rechtsstaatlicher Prinzipien vorexerziert. Der Film führt am Schicksal Joes die Labilität von Rechtsstaatlichkeit, Demokratie, Freiheit und menschlicher Zivilisation vor Augen. Die Masse, mit der sich Joe identifiziert hatte und in der das Potential zur Demokratie wie zur Demokratisierung von Wohlstand schlummert, erweist sich von ihrer Kehrseite her als alle Werte und Normen verkehrender und ignoranter Mob. ${ }^{19}$

Individuelles Geltungsbedürfnis und mangelndes Vertrauen in das Funktionieren des Staates (dieses erinnert an die zeitgenössischen faschistischen Positionen der Silver Shirt Legion und Black Legion $)^{20}$ stehen ebenso an der Wurzel dieses Übels wie das Verblassen der Kenntnis rechtsstaatlicher Prinzipien, so zeigt der Film auf der Handlungsebene und durch filmästhetische Mittel. Fritz Lang ist diese Erkenntnis so wichtig, dass er sie an die diskursive Oberfläche treten lässt und in

19. Vgl. die parallelen Darstellungen (selbst)zerstörerischer Massen in Langs früheren Filmen „Metropolis“ (1927) und „M“(1931).

20. S. dazu Schütze, Fritz Lang im Exil, S. 32. 
Tableaus kristallisiert. Tratschende Nachbarinnen machen sich durch angebliches Geheimwissen wichtig, die Gerüchteküche verbietet jede Frage nach dem Beweis einer Schuld, und beim Barbier zeigt sich die unter den Kunden verbreitete Unkenntnis der Verfassung - eine der wenigen Stellen, an denen Fritz Lang auch die eigene Perspektive als Zuwanderer mit reflektiert. So meint einer der Barbiere:

„Es ist unmöglich, ein Gesetz durchzubringen, das die freie Meinungsäußerung aufhebt, jedenfalls in Friedenszeiten.“ - Ein Kunde antwortet: „Das glaube ich nicht. Wer behauptet das denn?“ - Der Barbier antwortet: „Die Verfassung. [...] Sollten Sie mal lesen, Sie wären vielleicht überrascht. Ich musste sie lesen, um Amerikaner zu werden. Sie mussten sie nicht lesen, weil Sie hier geboren sind.“

Auch der Sheriff - zunächst als prinzipienstarke Figur gezeichnet - belehrt Hilfssheriffs und Menge über das rechtsstaatliche Procedere und appelliert an die Vernunft - aber reflexive Diskurse von Demokratie und Rechtsstaatlichkeit sind der choralen Stimme populistischer Kommunikationsformen und der Performanz der Masse schwerlich gewachsen. Gerücht statt Faktum, Sensationsgier und Spaßbedürfnis statt kritische Infragestellung, Impuls statt Reflexion: Das sind die Zutaten, die die Mob-Suppe zum Kochen bringen, und die dazu führen, dass der in Untersuchungshaft sitzende Joe im amerikanischen Durchschnittsort Strand zum Opfer der Lynchjustiz wird.

Fritz Lang zeichnet den das Gefängnis in Brand setzenden Mob als entfesselt: fratzenhaft, instinktgesteuert und bestialisch sind nun die, die zuvor im Zeichen gutbürgerlicher Zivilisation noch im Garten Bettlaken zum Trocknen aufhängten, sich beim Barbier den Bart schneiden ließen und sich selbst als „Stützen der Gesellschaft" bezeichnen. Selbst die staatlichen und politischen Funktionsträger versagen - situativ und aus unterschiedlichen Gründen. Das menschliche Antlitz des vom Feuer bedrohten, um sein Leben schreienden Joe, vermag keine Empathie bei der Masse auszulösen. Empathie und Reflexion sind Kompetenzen des Individuums - der Mob ist als solcher stumpf und gewissenlos. ${ }^{21}$

Die titelgebende „Fury“ ist jedoch nicht nur auf diesen lynchenden Mob bezogen, der getrieben ist von orgienhafter Lust, Sensationsgier, Euphorie. The Fury, blinde Wut, ist vielmehr die bestimmende und treibende Kraft für Joe. Denn er konnte durch einen Zufall dem Inferno seiner Zelle im letzten Moment entkommen und hat somit für alle unbemerkt überlebt. An diesem Wendepunkt des Films zeigt sich, warum Fritz Lang so viel zu Beginn des Films investierte, um seinen Protagonisten als zivilisierten regular guy aufzubauen: Umso radikaler erscheint dem Zuschauer nun seine Verwandlung zum Gegenteil seiner selbst ${ }^{22}$, seine Entfremdung und Befremdlichkeit. Seine Rachgier ist so stark, dass er selbst

21. Fritz Lang selbst formuliert: „Die Massen verlieren ihr Gewissen, wenn sie zusammenkommen; sie werden ein Mob und haben kein personales Gewissen mehr. Was in einem Lynchmord geschieht, ist Ausdruck eines Gefühls der Massen - sie sind keine fühlenden Individuen.“ [Übersetzung S.W.] Engl. Original: „Masses lose conscience when they are together; they become a mob and they have no personal conscience anymore. Things that happen during a riot are the expression of a mass feeling - they are no longer the feelings of individuals." (Peter Bogdanovich, Fritz Lang in America. London/ New York, Studio Vista, 1967, S. 31.)

22. Schütze verweist auf die Nähe der Figur zu Fritz Langs „Doktor Mabuse“. 
der geliebten Katherine sein Überleben verheimlicht und den halb geschmolzenen, mit Namen gravierten Liebesring als Beweismittel seines Todes vor Gericht auftauchen lässt, um die lynchende Masse wegen Mordes (und nicht nur wegen versuchten Mordes) vor Gericht bringen zu können - bei drohender Todesstrafe. Er hat sich selbst, sein identitätsfundierendes Netzwerk von Überzeugungen verloren: „Ich bin tot", diagnostiziert er treffend. Das ihn als regular guy definierende und stabilisierende Mythen-Netzwerk ${ }^{23}$ ist zerrissen, er ist sich durch den Verlust des Glaubens an American Dream, Fortschritt, Demokratie, Amerikanische Verfassung, Freiheit und menschliche Zivilisation selbst fremd geworden ${ }^{24}$ : Der Mob soll das Schicksal erleiden, das er ihm zugedacht hatte, und dafür instrumentalisiert er rechtsstaatliche Verfahren, verkehrt sie ins vormoderne Auge-um-Auge-Prinzip. Doch die Bewohner von Strand halten zusammen, niemand bricht sein Schweigen, der Beweis individualisierbarer Schuld scheint unmöglich.

\section{Der Gott aus der Maschine: die Kontrolle durch Medien und Gewissen}

Nun führt Fritz Lang eine neue mediale Macht als Deus ex machina ein: Die modernen Medien mit ihrer Filmtechnik. Die Journalisten vor Ort haben den Lynchmord ohne einzugreifen und in der Hoffnung auf sensationelle Bilder gefilmt, und ihr Material dient nicht nur dem sensationslustigen Publikum als Kinounterhaltung, sondern auf heimliches Geheiß Joes nun auch der Beweisführung. Nach aussichtslosen Zeugenverhören lässt die Anklage einen Filmprojektor mit Leinwand in den Gerichtssaal bringen, der an die Stelle der verweigerten mündlichen Zeugenaussage das bewegte Bild treten lässt. Mit Entsetzen sehen sich die Angeklagten mit ihrer unbezweifelbaren Schuld konfrontiert, immer wieder arretiert in Screenshots. Die neue scheinbare Evidenz der Filmaufnahme steht am Beginn des modernen Mythos Kino - und wird von Fritz Lang hier als Retter in höchster Not instrumentalisiert. ${ }^{25}$ Joe könnte nun seinen Rachezug zu Ende bringen und die 22 Beschuldigten auf das Schafott führen, und er wäre auch bereit dazu, gäbe es nicht den Urgrund des

23. Durch ihre Vernetzung können sich Mythen wechselseitig stabilisieren. Ihre scheinbar evidente Bedeutung erklingt dann mit ,choraler Stimme، und erlangt dadurch potenzierte Distribution und Intensität der Verankerung im kulturellen Gedächtnis. S. dazu Stephanie Wodianka, „Einleitung“, in dies., Juliane Ebert (Hrsg.), Inflation der Mythen? Zur Vernetzung und Stabilität eines modernen Phänomens, Bielefeld, transcript, 2016, S. 7-26.

24. Mythen bergen das Potential, individuelle und kollektive Überzeugungen miteinander zu vernetzen und damit ideologische Orientierungssysteme zu stabilisieren. S. dazu Stephanie Wodianka, „Mythos und tabula rasa: Poetik des Erzählens, Erinnerung und Gedächtnis, Fläche und Raum“, in Yves Bizeul, Stephanie Wodianka (Hrsg.), Mythos und tabula rasa. Narrationen und Denkformen absoluten Anfangs und totaler Auslöschung, Bielefeld, transcript, 2018, S. 25-40.

25. Bemerkenswert ist, dass das Kameraauge der anwesenden Reporter jene unsichtbare Überwachungspotenz übernimmt, die Mabuse in Langs Dr. Mabuse - der Spieler zugeschrieben wird. Zur Überwachungs-Macht Mabuses s. Martin Blumenthal-Barby, Der asymmetrische Blick. Film und Überwachung, Paderborn, Wilhelm Fink, 2016, S. 153-178. 
in Frage gestellten Mythen-Netzwerks: das moderne protestantische Gewissen ${ }^{26}$ als verinnerlichte Wert- und Norminstanz des sich selbst verantwortlichen Individuums, das Schuld als Selbstentfremdung definiert. Das moderne individuelle Gewissen, das der Film hier (neben moderner Filmtechnik) als Deus ex machina inszeniert, ist imprägniert von den Werten und Normen des Mythenclusters von American Dream, rechtsstaatlicher und demokratischer Verfassung, Fortschritt und Zivilisation. Fritz Lang stellt filmtechnisch versiert die Flucht Joes vor dem ihn verfolgenden Schuldgefühl dar. Das Gewissen Joes beurteilt die Entfremdung von seinem belief system schließlich als individuelle Schuld. ${ }^{27}$ Joe gibt seinen Racheplan auf und zeigt sich dem Gericht als Überlebender - die Angeklagten, die den Tod Joes wollten und ihn erreicht $\mathrm{zu}$ haben glauben, sind folglich nur noch wegen versuchten Mordes belastbar. Das Gewissen ist das Herzstück des regular guy, der im Film wiederum im Herzen des Mythenclusters positioniert wird. Ihm ist deshalb das zweite und letzte Glaubensbekenntnis Joes gewidmet, das er im Gerichtsaal spricht und das ein Bekenntnis des Glaubensverlusts ist. Er nennt die

\begin{abstract}
Dinge, die mir wichtig waren, lächerliche Dinge vielleicht, wie der Glaube an die Gerechtigkeit. Glaube daran, dass alle Menschen menschlich sind. Und der Stolz auf mein Land, von dem ich glaubte, dass es anders wäre als alle anderen. Das Gesetz weiß nicht, dass all diese Dinge an diesem Abend mit mir verbrannt sind. Aber ich konnte es nicht mit meinem Gewissen vereinbaren. Deshalb bin ich gekommen. Ich hätte nicht mit dieser Schuld weiterleben können. Ich hätte mich selbst verloren.
\end{abstract}

Am Ende seiner Rede bittet Joe seine Braut um eine Chance auf Wiedergutmachung, der Film endet - übrigens gegen den Widerstand Fritz Langs - mit einem innigen Kuss des Paares. Das individuelle Glück der Protagonisten, das zu Anfang des Films vor dem Schaufenster entworfen wird, scheint am Ende des Films wieder greifbar nahegekommen. Doch es bleibt stets bedroht, weil es - wie der Film zeigt - abhängig ist von der Gesamtstabilität eines belief systems, das anfällig ist, wenn man es nicht in seiner kollektiven, kulturellen Relevanz pflegt. Diese Pflege realisiert der Film Fritz Langs, der - wie zitiert - mehr sein will als Affirmation, sondern Orientierung gebender kultureller Richtungsweiser.

\title{
The Fury: Nibelungenklage der Moderne
}

Einerseits und bei unmittelbarer Betrachtung ist Fury ein Exilwerk, das sich auf die gesellschaftliche Realität des Exillandes konzentriert und mit dem Fritz Lang beweisen konnte, dass er in den USA angekommen war - und dass er in der Lage war, sich mit den neuen sozialen und politischen Kontexten kritisch auseinanderzusetzen ${ }^{28}$, ohne

26. Dieter Kittsteiner, Die Entstehung des modernen Gewissens, Frankfurt a. M., Insel Verlag, 1991, S. 159-165.

27. Dies nicht zuletzt durch den Appell Katherines, die - akustisch untermalt von Donnergroll - bekundet, sich im Falle der Durchsetzung des Racheplans von Joe lossagen zu müssen, weil er dann nicht mehr jener Joe sei, den sie liebe.

28. Dies sah Fritz Lang als eine der wichtigsten Aufgaben der Filmemacher an: „Die größte Verantwortung des Filmemachers ist es, seine Zeit zu reflektieren. Wenn die Menschen an die Zukunft glauben, muss 
das Publikum zu brüskieren. Bei einer stärker komparatistisch ausgerichteten Analyse zeigt sich andererseits und darüber hinaus, dass Lang mit diesem Film auch die Chance nutzte, an seine Erfolge vor dem Exil anzuschließen und damit neue, auch über den Horizont Amerikas hinausweisende Deutungspotentiale zu eröffnen, auch am eigenen mythe personnel als Regisseur zu arbeiten.

Dies gelingt ihm mit der Durchwirkung des Films Fury mit Referenzen auf den eingangs als mythische Erzählung über das eigene Fremde interpretierten Film Die Nibelungen, die bisher von der einschlägigen Forschung nicht gesehen wurden und die ich hier aufzeigen möchte. Der Überlebende Joe beharrt gegenüber seinen Brüdern explizit auf der rechtsstaatlichen Ahndung des Lynchmordes an ihm mit der Todesstrafe (,Sie sollen eine ordentliche Verhandlung in einem ordentlichen Gerichtssaal bekommen"), so wie Kriemhild ihren Bruder vergeblich aufgefordert hatte, über die Ermordung ihres Siegfried Gericht zu halten: „Ich rufe dich zum Gericht, König Gunther! Hagen Tronje erschlug mir den Gemahl!"“. Kriemhild war daraufhin mit einer Mauer des Schweigens konfrontiert, die die Ahndung des Verbrechens unmöglich machte (in Langs Die Nibelungen hatte es geheißen „Treue um Treue [...]! Seine Tat ist die unsere! Sein Los ist das unsere! Unsere Brust ist sein Schild“) - und auch in Fury betont Fritz Lang das kollektive Schweigen der Bewohner, die zusammenhalten und sich vor Gericht gegenseitig Alibis verschaffen. Bemerkenswert ist vor allem, dass Peter Lang Joe immer wieder äußern lässt „Ich bin tot / Ich bin ein Toter“ bzw. „all diese Dinge sind in jener Nacht mit mir verbrannt“, um den Verlust seines belief systems anzuzeigen, so wie Kriemhild gesprochen hatte „Ich starb, als Siegfried starb", als man an ihre Menschlichkeit appellierte. Wie Kriemhild und Siegfried in Die Nibelungen lebt das Protagonistenpaar Joe und Katherine im Zustand der Fernminne, und Fury wiederholt auch die Geschwisterkonstellation der Nibelungen. Wie die gutgläubige Kriemhild mit einem eingestickten Kreuz die Stelle im Gewand des Siegfried markiert hatte, an der der Unverwundbare verwundbar war, so wird auch Katherine Näharbeit an Joes zerrissener Jacke zum Zeichen, das ihn verrät. Wie Etzel in Die Nibelungen zunehmend menschlicher und Kriemhild zunehmend unmenschlicher erscheint, durchläuft auch Joe eine Entwicklung zum Unmenschlichen, während seine Brüder zunehmend anständiger werden. Und nicht zuletzt: Fritz Lang inszeniert den Lynchmord an Joe in deutlicher Referenz auf das von Kriemhild initiierte PalastInferno, mit dem sie ihre Gegner grausam vernichtete, und dessen Darstellung in Fritz Langs Die Nibelungen viel Raum zugestanden wird. Denn während das historische Lynchereignis in San José durch Erhängen der beiden Verdächtigen vollzogen wurde, entschied sich Fritz Lang in Fury für eine Brandschatzung des Gefängnisses durch den wütenden Mob, um die im Film angelegten Parallelen zu Die Nibelungen zu betonen. Zusätzliche filmästhetische Akzentuierung erfährt diese Parallele durch die Tatsache, dass Lang den Tonfilm Fury an dieser Stelle ,erstummen“ lässt, die InfernoSzene in Die Nibelungen und in Fury werden ohne Worte und mit weit geöffneten

der Filmemacher auch an sie glauben. Und wenn sie nach Wegen suchen, die Zukunft zu bewältigen, muss er Pfade bahnen.“ [Übersetzung S.W.] Engl. Original: „The highest responsability oft he film creator is to reflect his times. If the people believe in the future, the film creator must also believe in it. Andi $f$ they search for ways to achieve the future, he must chart the way.“ (Fritz Lang, „Happily Even After", The Penguin Film Review 5, 1948, S. 29.) 
Augen vom Lynchpublikum betrachtet. Und wie in Die Nibelungen gelingt es Fritz Lang in Fury, Mittelalter und Moderne über mythische Ikonen zu amalgamieren: Die rasende Brandschatzerin, die in Fury euphorisch ein brennendes Tuch schwenkt, um den Scheiterhaufen der Lynchtat zu entzünden, erscheint im Screenshot des im Gerichtssaal gezeigten Filmmaterials in der pervertierten Pose der Fackel haltenden Freiheitsstatue. Das vertraute Eigene zeigt sein fremdes, verdrängtes Gesicht.

Fritz Langs Fury ist in diesem Sinne eine neue Nibelungenklage unter den Vorzeichen der Moderne - und des Exils. Mit den Referenzen auf Die Nibelungen schließt der Regisseur seinen Film über die Fragilität und Bedrohtheit des amerikanischen belief systems an jene Filmästhetik an, mit der er das Mittelalter nicht als das vertraute Eigene, sondern mit orientalisierenden und exotisierenden Mitteln als die eigene Fremde zur Darstellung gebracht hatte. Fury zeigt, wie ein Kollektiv (die Bewohner von Strand) und wie ein Individuum (Joe) in die Gefahr geraten kann, sich von sich selbst und den eigenen Überzeugungen zu entfremden. Die wachsende Unvertrautheit und Entfremdung Amerikas von Gründungs- und Bestandsmythen wie Freiheit, Fortschritt, American Dream, Zivilisation und Verfassung wird von Fritz Lang in Fury durch den Rekurs auf Die Nibelungen als gefährliche zeitgenössische politische Gemengelage in den USA identifiziert, die zu Entwicklungen führen kann, die jenen des nationalsozialistischen Deutschland ähneln, vor denen er ins Exil geflohen war.

Die Nibelungen war für Fritz Lang kein exklusiv deutscher Mythos, sondern die mythische Erzählung von der Fremdheit eigener Mythen, die immer wieder neu anzueignen sind. Sein Exilland Amerika muss sich vor der Entfremdung vom Eigenen hüten und sich auf die mythische Figur des John Doe und dessen belief system besinnen, und auf die ethische Verantwortung des Films - so Langs filmischer und gesellschaftsanalytischer Appell mit Fury im Jahr 1936. ${ }^{29}$

29. Fury entstand im selben Jahr, in dem Fritz Lang Gründungsmitglied der „Hollywood Anti Nazi League" wurde - und damit manche Zweifel an seiner antifaschistischen Haltung aus dem Weg räumte. S. dazu Schütze, Fritz Lang im Exil, S. 38-46. 
